\title{
Oscillation of a logistic equation with delay and diffusion
}

\author{
by Sheng Li XIE (Jinzhou) and Sui Sun Cheng (Hsinchu)
}

Abstract. This paper establishes oscillation theorems for a class of functional parabolic equations which arises from logistic population models with delays and diffusion.

1. Introduction. This paper is concerned with a parabolic differential equation with delays which can be used to model the dynamical behavior of a population density governed by a logistic law with time delays and spatial diffusion. Assuming a positive stationary state of the population density, we are interested to seek conditions under which every possible evolution oscillates about it. Similar problems have been dealt with in a few recent studies (see e.g. [2, Chapter 6], [8, 6, 4, 7], [5, Chapter 4] and [3]). The literature is, however, quite limited.

Let $\tau_{i}(t), 1 \leq i \leq n$, be positive continuous functions defined on $[0, \infty)$ such that $\tau=\max _{i}\left\{\max _{t} \tau_{i}(t)\right\}<\infty$. Let $\Omega$ be a bounded domain in $\mathbb{R}^{m}$ with a smooth boundary $\partial \Omega$ and let $\nu$ be the outward unit normal vector. As usual, let $\Delta$ be the Laplacian:

$$
\Delta \equiv \frac{\partial^{2}}{\partial x_{1}^{2}}+\ldots+\frac{\partial^{2}}{\partial x_{m}^{2}}
$$

Consider the following functional parabolic differential equation:

$$
\text { (1) } \frac{\partial u(x, t)}{\partial t}=d(t) \Delta u(x, t)+c(t) u(x, t)\left\{a(t)-\sum_{i=1}^{n} b_{i}(t) u\left(x, t-\tau_{i}(t)\right)\right\} \text {, }
$$

where $(x, t) \in \Omega \times(0, \infty), a(t), c(t), d(t), b_{1}(t), \ldots, b_{n}(t)$ are positive, bounded and continuous functions on $[0, \infty)$ and $0<d_{0} \leq d(t), 0<b_{0} \leq c(t) b_{i}(t)$ for some $i \in\{1, \ldots, n\}$ on $[0, \infty)$. We will consider boundary conditions of the

1991 Mathematics Subject Classification: 35K22, 35K99.

Key words and phrases: oscillation theorem, functional parabolic differential equation, logistic equation. 
form

$$
\begin{aligned}
& \frac{\partial u(x, t)}{\partial \nu}=0, \quad(x, t) \in \partial \Omega \times(0, \infty), \\
& u(x, t)=\phi(x, t), \quad(x, t) \in \Omega \times[-\tau, 0],
\end{aligned}
$$

where $\phi(x, t)$ is a non-negative and non-trivial continuous function.

Existence and uniqueness theorems for solutions of (1)-(3) follow from the existence of a unique "heat kernel" $g(x, t, \xi, \mu)$ associated with the differential operator $L[u]=u_{t}-d(t) u_{x x}$ and the boundary condition (2). By means of this kernel (1)-(3) can be transformed into an integral equation which is well posed and can be solved by the "method of steps". For details, see Kreith and Ladas [7], and [9, 1, 10]. In what follows, by a solution of (1)(3), we mean a function $u(x, t)$ which is continuously differentiable on the closure of $\Omega \times[-\tau, \infty)$ and twice continuously differentiable on $\Omega \times[-\tau, \infty)$.

We will further assume that there is a positive constant $u^{*}$ such that

$$
\sum_{i=1}^{n} b_{i}(t) u^{*}=a(t), \quad t \geq 0,
$$

so that $u(x, t)=u^{*}$ is a stationary solution of $(1)$.

Let $u(x, t)$ be a real continuous function defined on $\bar{\Omega} \times\left[t_{0}, \infty\right)$. Suppose there is some $T \geq t_{0}$ such that $u(x, t)>0$ on $\Omega \times[T, \infty)$. Then $u(x, t)$ is said to be eventually positive. An eventually negative $u(x, t)$ is similarly defined. The function $u(x, t)$ is said to be oscillatory on $\Omega \times\left[t_{0}, \infty\right)$ if it is neither eventually positive nor eventually negative. Equation (1) is said to be oscillatory about $u^{*}$ if for every positive solution $u(x, t)$ of $(1)$ which satisfies the boundary conditions (2) and (3), the function $u(x, t)-u^{*}$ is oscillatory on $\Omega \times[-\tau, \infty)$.

2. Non-existence criteria. We first derive a necessary condition for the existence of a solution $u(x, t)$ of $(1)$ such that $u(x, t)-u^{*}$ is eventually positive.

Lemma 1. Suppose (1)-(3) has a solution $u(x, t)$ such that $u(x, t)-u^{*}$ is eventually positive. Then the first order delay differential inequality

$$
y^{\prime}(t) \leq-\sum_{i=1}^{n} u^{*} c(t) b_{i}(t) y\left(t-\tau_{i}(t)\right)
$$

has an eventually positive solution.

Proof. Suppose there is a positive number $t_{1}$ such that $u(x, t)-u^{*}>0$ on $\Omega \times\left[t_{1}, \infty\right)$. For convenience, let $w(x, t)=u(x, t)-u^{*}$. Then from (1) 
and (4), we have

(6) $\frac{\partial w(x, t)}{\partial t}=d(t) \Delta w(x, t)-c(t)\left[w(x, t)+u^{*}\right] \sum_{i=1}^{n} b_{i}(t) w\left(x, t-\tau_{i}(t)\right)$.

Integrate both sides of (6) with respect to $x$ over $\Omega$ to obtain

$$
\begin{aligned}
\frac{d}{d t} \int_{\Omega} w(x, t) d x= & d(t) \int_{\Omega} \Delta w(x, t) d x \\
& -c(t) \int_{\Omega}\left[w(x, t)+w^{*}\right] \sum_{i=1}^{n} b_{i}(t) w\left(x, t-\tau_{i}(t)\right) d x
\end{aligned}
$$

By the Green formula and the boundary condition (2), we obtain

$$
\int_{\Omega} \Delta w(x, t) d x=\int_{\partial \Omega} \frac{\partial w(x, t)}{\partial \nu} d s=0 .
$$

Pick a number $t_{2}>t_{1}+\tau$. Then $w(x, t)>0$ and $w\left(x, t-\tau_{i}(t)\right)>0$ for $(x, t) \in \Omega \times\left[t_{2}, \infty\right)$. In view of (7) and (8), we have

$$
\frac{d}{d t} \int_{\Omega} w(x, t) d x \leq-c(t) \int_{\Omega} u^{*} \sum_{i=1}^{n} b_{i}(t) w\left(x, t-\tau_{i}(t)\right) d x, \quad t \geq t_{2} .
$$

We have thus shown that

$$
y(t)=\int_{\Omega} w(x, t) d x, \quad t \geq t_{2},
$$

is an eventually positive solution of (5). The proof is complete.

Next we derive a necessary condition for the existence of a positive solution $u(x, t)$ of $(1)$ such that $u(x, t)-u^{*}$ is eventually negative.

LEMma 2. Suppose (1)-(3) has a positive solution $u(x, t)$ such that $u(x, t)$ $-u^{*}$ is eventually negative. Then for any $\mu \in(0,1)$, the first order delay differential inequality

$$
y^{\prime}(t) \leq-\sum_{i=1}^{n} u^{*} \mu c(t) b_{i}(t) y\left(t-\tau_{i}(t)\right)
$$

has an eventually positive solution.

Proof. Suppose there is a positive number $t_{1}$ such that $u(x, t)-u^{*}<0$ for $(x, t) \in \Omega \times\left[t_{1}, \infty\right)$. Let

$$
p(x, t)=\ln \left(\frac{u(x, t)}{u^{*}}\right)<0, \quad(x, t) \in \Omega \times\left[t_{1}, \infty\right) .
$$


We assert that

$$
y(t)=\int_{\Omega}-p(x, t) d x
$$

is an eventually positive solution of (9). To prove this, note first that from (1) and (4) we have

$$
\text { (10) } \begin{aligned}
\frac{\partial p(x, t)}{\partial t} & =d(t) e^{-p(x, t)} \Delta e^{p(x, t)}+c(t)\left\{a(t)-\sum_{i=1}^{n} b_{i}(t) u^{*} e^{p\left(x, t-\tau_{i}(t)\right)}\right\} \\
& =d(t) e^{-p(x, t)} \Delta e^{p(x, t)}-u^{*} c(t) \sum_{i=1}^{n} b_{i}(t)\left\{e^{p\left(x, t-\tau_{i}(t)\right)}-1\right\} .
\end{aligned}
$$

Integrate the above equality with respect to $x$ over $\Omega$ to obtain

$$
\begin{aligned}
\frac{d}{d t} \int_{\Omega} p(x, t) d x= & d(t) \int_{\Omega} e^{-p(x, t)} \Delta e^{p(x, t)} d x \\
& \quad-u^{*} c(t) \sum_{i=1}^{n} b_{i}(t) \int_{\Omega}\left\{e^{p\left(x, t-\tau_{i}(t)\right)}-1\right\} d x .
\end{aligned}
$$

Since

(12) $\frac{\partial}{\partial \nu}\left\{e^{ \pm p(x, t)}\right\}= \pm e^{ \pm p(x, t)} \frac{\partial p(x, t)}{\partial \nu}, \quad(x, t) \in \partial \Omega \times\left[t_{1}, \infty\right)$,

(13) $\left.\frac{\partial p(x, t)}{\partial \nu}\right|_{\partial \Omega}=\left.\frac{1}{u(x, t)} \frac{\partial u(x, t)}{\partial \nu}\right|_{\partial \Omega}=0$,

and

$$
e^{p(x, t)} \Delta e^{-p(x, t)}=|\nabla p(x, t)|^{2}-\Delta p(x, t), \quad(x, t) \in \Omega \times\left[t_{1}, \infty\right),
$$

we obtain

$$
\begin{aligned}
\int_{\Omega} e^{-p(x, t)} \Delta e^{p(x, t)} d x & =\int_{\Omega} e^{p(x, t)} \Delta e^{-p(x, t)} d x \\
& =\int_{\Omega}|\nabla p(x, t)|^{2} d x-\int_{\Omega} \Delta p(x, t) d x \\
& =\int_{\Omega}|\nabla p(x, t)|^{2} d x-\int_{\partial \Omega} \frac{\partial p(x, t)}{\partial \nu} d s \\
& =\int_{\Omega}|\nabla p(x, t)|^{2} d x .
\end{aligned}
$$

From (11) we also obtain 
(15) $\frac{d}{d t} \int_{\Omega}-p(x, t) d x$

$$
\begin{aligned}
& =-d(t) \int_{\Omega}|\nabla p(x, t)|^{2} d x+u^{*} c(t) \sum_{i=1}^{n} b_{i}(t) \int_{\Omega}\left\{e^{p\left(x, t-\tau_{i}(t)\right)}-1\right\} d x \\
& \leq u^{*} c(t) \sum_{i=1}^{n} b_{i}(t) \int_{\Omega}\left\{e^{p\left(x, t-\tau_{i}(t)\right)}-1\right\} d x
\end{aligned}
$$

To complete the proof, it suffices to show that for any $\mu \in(0,1)$, there is some $T_{0}$ such that

$$
e^{p\left(x, t-\tau_{i}(t)\right)}-1 \leq \mu p\left(x, t-\tau_{i}(t)\right), \quad t \geq T_{0} .
$$

In order to do this, pick $t_{2}>t_{1}+\tau$ so that $p(x, t)<0$ and $p\left(x, t-\tau_{i}(t)\right)<$ 0 for $(x, t) \in \Omega \times\left[t_{2}, \infty\right)$. For $(x, t) \in \Omega \times\left[t_{2}, \infty\right)$, consider the positive functional $V$ defined by

$$
V[p](t)=u^{*} \int_{\Omega} \int_{0}^{p(x, t)}\left(e^{y}-1\right) d y d x .
$$

By (10) and (12)-(14), the derivative of $V$ with respect to (1) satisfies

$$
\begin{aligned}
\frac{d V}{d t}= & u^{*} \int_{\Omega}\left(e^{p(x, t)}-1\right) \frac{\partial p(x, t)}{\partial t} d x \\
= & u^{*} d(t) \int_{\Omega} \Delta e^{p(x, t)} d x-u^{*} d(t) \int_{\Omega} e^{-p(x, t)} \Delta e^{p(x, t)} d x \\
& -\left(u^{*}\right)^{2} c(t) \sum_{i=1}^{n} b_{i}(t) \int_{\Omega}\left\{e^{p(x, t)}-1\right\}\left\{e^{p\left(x, t-\tau_{i}(t)\right)}-1\right\} d x \\
\leq & -u^{*} d(t) \int_{\Omega}|\nabla p(x, t)|^{2} d x \\
& -\left(u^{*}\right)^{2} c(t) \sum_{i=1}^{n} b_{i}(t) \int_{\Omega}\left\{e^{p(x, t)}-1\right\}\left\{e^{p\left(x, t-\tau_{i}(t)\right)}-1\right\} d x
\end{aligned}
$$

Note that from (1),

$$
\begin{aligned}
\frac{d}{d t} \int_{\Omega} u(x, t) d x= & \int_{\Omega} \frac{\partial u(x, t)}{\partial t} d x \\
= & \int_{\Omega} d(t) \Delta u(x, t) d x \\
& +\int_{\Omega} u(x, t) c(t) \sum_{i=1}^{n} b_{i}(t)\left(u^{*}-u\left(x, t-\tau_{i}(t)\right)\right) d x
\end{aligned}
$$




$$
=\int_{\Omega} u(x, t) c(t) \sum_{i=1}^{n} b_{i}(t)\left(u^{*}-u\left(x, t-\tau_{i}(t)\right)\right) d x \geq 0,
$$

thus

$$
\int_{\Omega}\left(u(x, t)-u\left(x, t-\tau_{i}(t)\right)\right) d x \geq 0
$$

which implies

$$
\begin{aligned}
& \int_{\Omega}\left\{e^{p(x, t)}-1\right\}\left\{e^{p\left(x, t-\tau_{i}(t)\right)}-1\right\} d x \\
& =\int_{\Omega}\left\{e^{p(x, t)}-1\right\}^{2} d x+\int_{\Omega}\left\{e^{p(x, t)}-1\right\}\left\{e^{p\left(x-\tau_{i}(t)\right)}-e^{p(x, t)}\right\} d x \\
& =\int_{\Omega}\left\{e^{p(x, t)}-1\right\}^{2} d x+\frac{1}{\left(u^{*}\right)^{2}} \int_{\Omega}\left(u(x, t)-u^{*}\right)\left(u\left(x, t-\tau_{i}(t)\right)-u(x, t)\right) d x \\
& \geq \int_{\Omega}\left\{e^{p(x, t)}-1\right\}^{2} d x
\end{aligned}
$$

by the first mean value theorem for integrals. As a consequence, we see that

$$
\begin{aligned}
\frac{d V}{d t} \leq & -u^{*} d(t) \int_{\Omega}|\nabla p(x, t)|^{2} d x \\
& -\left(u^{*}\right)^{2} c(t) \sum_{i=1}^{n} b_{i}(t) \int_{\Omega}\left\{e^{p(x, t)}-1\right\}^{2} d x \\
= & -u^{*} d(t) \int_{\Omega}|\nabla p(x, t)|^{2} d x \\
& -\left(u^{*}\right)^{2} c(t) \sum_{i=1}^{n} b_{i}(t) \int_{\Omega}\left\{u(x, t)-u^{*}\right\}^{2} d x
\end{aligned}
$$

for $t \geq t_{2}$. Integrate both sides of (16), and recall the assumptions that $0<d_{0} \leq d(t)$ and $0<b_{0} \leq b_{i}(t) c(t)$, to obtain

$$
\begin{aligned}
V\left(t_{2}\right) \geq & V(t)+u^{*} d_{0} \int_{t_{2}}^{t} \int_{\Omega}|\nabla p(x, t)|^{2} d x d y \\
& +\left(u^{*}\right)^{2} b_{0} \int_{t_{2}}^{t} \int_{\Omega}\left\{u(x, y)-u^{*}\right\}^{2} d x d y .
\end{aligned}
$$

Hence, by writing $\left\{\int_{\Omega}|\cdot|^{2} d x\right\}^{1 / 2}=\|\cdot\|$, we have

$$
\int_{t_{2}}^{\infty} \int_{\Omega}\left\{u(x, y)-u^{*}\right\}^{2} d x d y=\int_{t_{2}}^{\infty}\left\|u(x, y)-u^{*}\right\|^{2} d y<\infty
$$


and

$$
\int_{t_{2}}^{\infty} \int_{\Omega}|\nabla p(x, y)|^{2} d x d y=\int_{t_{2}}^{\infty}\|\nabla p(x, y)\|^{2} d y<\infty,
$$

so that $\left\|u(x, y)-u^{*}\right\| \in L_{1}(0, \infty)$ and $\|\nabla p(x, t)\|^{2} \in L_{1}(0, \infty)$. But from the assumption that $u(x, t)<u^{*}$ for $(x, t) \in \Omega \times\left[t_{1}, \infty\right)$ we have

$$
\frac{1}{\left(u^{*}\right)^{2}}\|\nabla u(x, t)\|^{2} \leq\|\nabla p(x, t)\|^{2}
$$

so that $\|\nabla u(x, t)\|^{2} \in L_{1}(0, \infty)$. Now

$$
\begin{aligned}
\frac{d}{d t} \| \nabla & u(x, t) \|^{2} \\
= & \left(\frac{\partial u(x, t)}{\partial t},-\Delta u(x, t)\right) \\
= & -d(t)\|\Delta u(x, t)\|^{2} \\
& +\left(\nabla\left\{c(t) u(x, t)\left[a(t)-\sum_{i=1}^{n} b_{i}(t) u\left(x, t-\tau_{i}(t)\right)\right]\right\}, \nabla u(x, t)\right) \\
\leq & -d_{0}\|\Delta u(x, t)\|^{2}+c a\|\nabla u(x, t)\|^{2} \\
& +u^{*} c \sum_{i=1}^{n} b_{i}\left\|\nabla u\left(x, t-\tau_{i}(t)\right)\right\|\|\nabla u(x, t)\|,
\end{aligned}
$$

where $|a(t)| \leq a,|c(t)| \leq c,\left|b_{i}(t)\right| \leq b_{i}$ for $t \geq 0$ (recall that $a(t), c(t)$ and $b_{i}(t)$ are bounded).

Integrate both sides of (17) from $t=t_{2}$ to $t=T>t_{2}$ to obtain

$$
\begin{aligned}
& \|\nabla u(x, T)\|^{2}-\left\|\nabla u\left(x, t_{2}\right)\right\|+d_{0} \int_{t_{2}}^{T}\|\Delta u(x, t)\|^{2} d t \\
\leq & c a \int_{t_{2}}^{T}\|\nabla u(x, t)\|^{2} d t+u^{*} c \sum_{i=1}^{n} b_{i} \int_{t_{2}}^{T}\left\|\nabla u\left(x, t-\tau_{i}(t)\right)\right\|\|\nabla u(x, t)\| d t \\
\leq & c a \int_{t_{2}}^{T}\|\nabla u(x, t)\|^{2} d t \\
& +u^{*} c \sum_{i=1}^{n} b_{i}\left\{\int_{t_{2}}^{T}\left\|\nabla u\left(x, t-\tau_{i}(t)\right)\right\|^{2} d t\right\}^{1 / 2}\left\{\int_{t_{2}}^{T}\|\nabla u(x, t)\|^{2} d t\right\}^{1 / 2} .
\end{aligned}
$$

We may now infer from $\|\nabla u(x, t)\|^{2} \in L_{1}(0, \infty)$ and the above inequality that $\|\Delta u(x, t)\|^{2} \in L_{1}(0, \infty)$ and $\|\nabla u(x, t)\|^{2}$ is bounded on $\left(t_{2}, \infty\right)$. 
In a similar fashion, we obtain

$$
\begin{aligned}
& \int_{t_{2}}^{T}\left|\frac{d}{d t}\|\nabla u(x, t)\|^{2}\right| d t \\
& \leq d_{0} \int_{t_{2}}^{T}\|\Delta u(x, t)\|^{2} d t+c a \int_{t_{2}}^{T}\|\nabla u(x, t)\|^{2} d t \\
& \quad+u^{*} c \sum_{i=1}^{n} b_{i}\left\{\int_{t_{2}}^{T}\left\|\nabla u\left(x, t-\tau_{i}(t)\right)\right\|^{2} d t\right\}^{1 / 2}\left\{\int_{t_{2}}^{T}\|\nabla u(x, t)\|^{2} d t\right\}^{1 / 2}
\end{aligned}
$$

Since $\|\nabla u(x, t)\|^{2} \in L_{1}(0, \infty)$ and $\|\Delta u(x, t)\|^{2} \in L_{1}(0, \infty)$, we may deduce the fact that $(d / d t)\|\nabla u(x, t)\|^{2} \in L_{1}(0, \infty)$.

We now make a few more deductions. First, from $\|\nabla u(x, t)\|^{2} \in L_{1}(0, \infty)$ and $(d / d t)\|\nabla u(x, t)\|^{2} \in L_{1}(0, \infty)$, by integrating $(d / d t)\|\nabla u(x, t)\|^{2}$ from a large but fixed number to infinity, we see that as $t$ tends to infinity, $\|\nabla u(x, t)\|^{2}$ tends to a constant which must be zero, i.e.

$$
\lim _{t \rightarrow \infty} \int_{\Omega}|\nabla u(x, t)|^{2} d t=\lim _{t \rightarrow \infty}\|\nabla u(x, t)\|^{2}=0 .
$$

Furthermore, since

$$
\begin{aligned}
& \frac{1}{2} \frac{d}{d t}\left\|u(x, t)-u^{*}\right\|^{2} \\
& =\int_{\Omega}\left(u(x, t)-u^{*}\right) \frac{\partial u(x, t)}{\partial t} d x \\
& =d(t) \int_{\Omega}\left(u(x, t)-u^{*}\right) \Delta u(x, t) d x \\
& \quad-\int_{\Omega}\left(u(x, t)-u^{*}\right) c(t) u(x, t) u(x, t) \sum_{i=1}^{n} b_{i}(t)\left(u\left(x, t-\tau_{i}(t)\right)-u^{*}\right) d x
\end{aligned}
$$

we have

$$
\begin{aligned}
\mid \frac{1}{2} \frac{d}{d t} \| u(x, t)- & u^{*} \|^{2} \mid \\
\leq & d \int_{\Omega}\left|u(x, t)-u^{*}\right| \cdot|\Delta u(x, t)| d x \\
& +c \sum_{i=1}^{n} b_{i} u^{*} \int_{\Omega}\left|u(x, t)-u^{*}\right| \cdot\left|u\left(x, t-\tau_{i}(t)\right)-u^{*}\right| d x
\end{aligned}
$$


and

$$
\begin{aligned}
\int_{t_{3}}^{T} \mid \frac{1}{2} \frac{d}{d t} \| u(x, t) & -u^{*} \|^{2} \mid d t \\
\leq & d\left\{\int_{t_{3}}^{T}\|\Delta u(x, t)\|^{2} d t\right\}^{1 / 2}\left\{\int_{t_{3}}^{T}\left\|u(x, t)-u^{*}\right\|^{2} d t\right\}^{1 / 2} \\
& +c u^{*} \sum_{i=1}^{n} b_{i}\left\{\int_{t_{3}}^{T}\left\|u(x, t)-u^{*}\right\|^{2} d t\right\}^{1 / 2} \\
& \times\left\{\int_{t_{3}}^{T}\left\|u\left(x, t-\tau_{i}(t)\right)-u^{*}\right\|^{2} d t\right\}^{1 / 2}
\end{aligned}
$$

As a consequence, we have

$$
\frac{d}{d t}\left\|u(x, t)-u^{*}\right\| \in L_{1}(0, \infty)
$$

in view of the previously shown facts that $\|\Delta u(x, t)\|^{2} \in L_{1}(0, \infty)$ and $\left\|u(x, t)-u^{*}\right\|^{2} \in L_{1}(0, \infty)$. By integrating $(d / d t)\left\|u(x, t)-u^{*}\right\|$ from a large but fixed number to infinity, we see that as $t$ tends to infinity, $\left\|u(x, t)-u^{*}\right\|$ tends to a constant which must be zero, i.e.

$$
\lim _{t \rightarrow \infty}\left\|u(x, t)-u^{*}\right\|=0 \text {. }
$$

Next, from $u(x, t)<u^{*}$ for $t>t_{2}$ and the boundedness of $\|\nabla u(x, t)\|$ on $\left(t_{2}, \infty\right)$, we see that $\left\|u(x, t)-u^{*}\right\|_{\infty}$ and $\|\nabla u(x, t)\|_{\infty}$ are bounded (where $\left.\|w\|_{\infty}=\operatorname{ess} \sup |w(x)|\right)$; then from the inequality

$$
\|w\|_{\sigma} \leq\|w\|_{\infty}^{(\sigma-2) / \sigma}\|w\|_{2}^{2 / \sigma} \quad \text { for all } \sigma \geq 2
$$

and (19) as well as (20), we obtain

$$
\lim _{t \rightarrow \infty}\left\|u(x, t)-u^{*}\right\|_{\sigma}=\lim _{t \rightarrow \infty}\|\nabla u(x, t)\|_{\sigma}=0, \quad \sigma>m .
$$

Next, from the Sobolev inequality, we obtain

$$
\begin{aligned}
& \left\|u(x, t)-u^{*}\right\|_{\infty} \\
& \quad \leq M(\Omega, m, \sigma)\left\{\left\|u(x, t)-u^{*}\right\|_{\sigma}+\left\|\nabla\left(u(x, t)-u^{*}\right)\right\|_{\sigma}\right\},
\end{aligned}
$$

where $M(\Omega, m, \sigma)$ is some positive constant.

Finally, from (21) and (22), we see that $\left\|u(x, t)-u^{*}\right\|_{\infty} \rightarrow 0$ as $t \rightarrow \infty$, so that

which implies

$$
\lim _{t \rightarrow \infty} u(x, t)=u^{*} \quad \text { uniformly in } x \in \bar{\Omega},
$$

$$
\lim _{t \rightarrow \infty} p(x, t)=0 \quad \text { uniformly in } x \in \bar{\Omega} .
$$


Now for any $t>t_{2}$ and $t_{3}>t_{2}$,

$$
e^{p\left(x, t-\tau_{i}(t)\right)}-e^{p\left(x, t_{3}\right)}=\left\{p\left(x, t-\tau_{i}(t)\right)-p\left(x, t_{3}\right)\right\} e^{p\left(x, \eta_{i}(t)\right)},
$$

where $e^{p\left(x, \eta_{i}(t)\right)} \rightarrow 1$ as $t \rightarrow \infty$. Thus for any $\mu \in(0,1)$, we can find $t_{4}$ such that

which implies

$$
\mu<e^{p\left(x, \eta_{i}(t)\right)}<1, \quad t \geq t_{4},
$$

$$
e^{p\left(x, t-\tau_{i}(t)\right)}-1 \leq \mu p\left(x, t-\tau_{i}(t)\right), \quad t \geq t_{4},
$$

as required. The proof is complete.

3. Oscillation criteria. In the last section, we have established necessary conditions for the existence of eventually positive or negative solutions. These conditions are related to delay differential inequalities. In order to obtain oscillation theorems, we only need conditions under which these inequalities do not have eventually positive solutions. Such a technique for obtaining oscillation theorems is standard (see for example [2, Chapter 6] or [7]). For illustration, we now mention several examples.

LEMma 3 (Ladas and Stavroulakis [8]; see also [2, p. 199]). Let $\sigma_{1}, \ldots, \sigma_{k}$ be positive constants and let $q_{1}(t), \ldots, q_{k}(t)$ be positive continuous functions on $\left[t_{0}, \infty\right)$. Suppose

$$
\liminf _{t \rightarrow \infty} \int_{t-\sigma_{i} / 2}^{t} q_{i}(s) d s>0, \quad 1 \leq i \leq k
$$

and

$$
\liminf _{t \rightarrow \infty} \int_{t-\sigma_{i}}^{t} q_{i}(s) d s>\frac{1}{e} \quad \text { for at least one } i \in\{1, \ldots, k\} .
$$

Then the differential inequality

$$
x^{\prime}(t)+\sum_{i=1}^{k} q_{i}(t) x\left(t-\sigma_{i}\right) \leq 0
$$

has no eventually positive solutions.

In view of Lemmas 1,2 and 3, we obtain the following oscillation theorem.

THEOREM 1. Suppose $\tau_{1}, \ldots, \tau_{n}$ are positive constants and $a(t), c(t)$, $d(t), b_{1}(t), \ldots, b_{n}(t)$ are bounded functions on $[0, \infty)$ such that for $t \geq 0$, $0<d_{0} \leq d(t)$ and $0<b_{0} \leq c(t) b_{i}(t)$ for some $i \in\{1, \ldots, n\}$. Suppose $u^{*}$ is a positive constant such that (4) holds. If

$$
\liminf _{t \rightarrow \infty} \int_{t-\tau_{i} / 2}^{t} c(s) b_{i}(s) d s>0, \quad 1 \leq i \leq n
$$


and

$$
\liminf _{t \rightarrow \infty} \int_{t-\tau_{i}}^{t} c(s) b_{i}(s) d s>\frac{1}{e} \quad \text { for at least one } i \in\{1, \ldots, n\} .
$$

Then equation (1) is oscillatory about $u^{*}$.

A result of Hunt and Yorke [6] states that if $\tau_{i}(t)$ and $q_{i}(t), 1 \leq i \leq n$, are positive continuous functions on $[0, \infty)$ such that

$$
\max _{i} \max _{t} \tau_{i}(t)<\infty
$$

and if

$$
\liminf _{t \rightarrow \infty} \sum_{i=1}^{n} q_{i}(t) \tau_{i}(t)>\frac{1}{e}
$$

then all solutions of

$$
x^{\prime}(t)+\sum_{i=1}^{n} q_{i}(t) x\left(t-\tau_{i}(t)\right)=0
$$

must oscillate. Another result in [5, Corollary 3.2.2] states that under the same assumptions on $\tau_{i}(t)$ and $q_{i}(t)$ as above, a necessary and sufficient condition for all solutions of (25) to oscillate is that the inequality

$$
x^{\prime}(t)+\sum_{i=1}^{n} q_{i}(t) x\left(t-\tau_{i}(t)\right) \leq 0
$$

does not have any eventually positive solution. In view of these two results and our Lemmas 1 and 2, we obtain the following result.

Theorem 2. Suppose $a(t), c(t), d(t), b_{1}(t), \ldots, b_{n}(t)$ and $\tau_{1}(t), \ldots, \tau_{n}(t)$ are positive bounded continuous functions on $[0, \infty)$ such that $(24)$ holds and for $t \geq 0,0<d_{0} \leq d(t)$ and $0<b_{0} \leq c(t) b_{i}(t)$ for some $i \in\{1, \ldots, n\}$. Suppose $u^{*}$ is a positive constant such that (4) holds. If

$$
\liminf _{t \rightarrow \infty} c(t) \sum_{i=1}^{n} b_{i}(t) \tau_{i}(t) u^{*}>\frac{1}{e}
$$

then equation (1) is oscillatory about $u^{*}$.

Other results can be obtained from corresponding oscillation theorems for equation (25), which can be found, among others, in [5, Section 3.4].

\section{References}

[1] A. Ardito and P. Ricciardi, Existence and regularity for linear delay partial differential equations, Nonlinear Anal. 4 (1980), 411-414. 
[2] D. D. Bainov and D. P. Mishev, Oscillation Theory for Neutral Differential Equations with Delay, Adam Hilger, Bristol, 1991.

[3] K. Gopalsamy, M. R. S. Kulenovic and G. Lad as, Time lags in a "food limited" population model, Appl. Anal. 31 (1988), 225-237.

[4] - , - - Oscillations of a system of delay logistic equations, J. Math. Anal. Appl. 146 (1990), 192-202.

[5] I. Györi and G. Lad as, Oscillation Theory of Delay Differential Equations, Clarendon Press, Oxford, 1991.

[6] B. R. Hunt and J. A. Yorke, When all solutions of $x^{\prime}=-\sum q_{i}(t) x\left(t-T_{i}(t)\right)$ oscillate, J. Differential Equations 53 (1984), 139-145.

[7] K. Kreith and G. Ladas, Allowable delays for positive diffusion processes, Hiroshima Math. J. 15 (1985), 437-443.

[8] G. Ladas and I. P. Stavroulakis, On delay differential inequalities of first order, Funkcial. Ekvac. 25 (1982), 105-113.

[9] C. C. Travis and G. F. Webb, Existence and stability for partial functional differential equations, Trans. Amer. Math. Soc. 200 (1974), 395-418.

[10] J. Turo, Generalized solutions of mixed problems for quasilinear hyperbolic systems of functional partial differential equations in the Schauder canonic form, Ann. Polon. Math. 50 (1989), 157-183.

DEPARTMENT OF MATHEMATICS JINZHOU TEACHER'S COLLEGE JINZHOU, HUBEI 434100 P.R. CHINA
DEPARTMENT OF MATHEMATICS TSING HUA UNIVERSITY HSINCHU 30043 TAIWAN, R.O.C. E-mail: SSCHENG@MATH.NTHU.EDU.TW 